Linguistique, littérature, didactique

\title{
La rétroaction négative en classe de grammaire
} Enjeux démocratiques

Negative Feedback in Grammar Class. Democracy at Stake

\section{Lionel Meinertzhagen}

\section{(2) OpenEdition}

Journals

\section{Édition électronique}

URL : http://journals.openedition.org/pratiques/2763

DOI : 10.4000/pratiques.2763

ISSN : 2425-2042

Éditeur

Centre de recherche sur les médiations (CREM)

\section{Référence électronique}

Lionel Meinertzhagen, «La rétroaction négative en classe de grammaire », Pratiques [En ligne], 167-168 | 2015, mis en ligne le 01 avril 2016, consulté le 01 mai 2019. URL : http:// journals.openedition.org/pratiques/2763; DOI : 10.4000/pratiques.2763

Ce document a été généré automatiquement le 1 mai 2019.

(c) Tous droits réservés 


\section{La rétroaction négative en classe de grammaire}

Enjeux démocratiques

Negative Feedback in Grammar Class. Democracy at Stake

\section{Lionel Meinertzhagen}

\section{Introduction}

1 Versé depuis 2009 dans l'observation de classes de grammaire au primaire et au secondaire en Belgique francophone dans le cadre de la recherche Gramm-R Scola, ce qui a retenu notre attention ici ne relève pas directement des objectifs de celle-ci, même si elle s'en trouve confortée. Initiée et toujours dirigée par Dan Van Raemdonck (2004-2013), elle peut être divisée en quatre grandes phases depuis 2003 : une d'analyse des problèmes, besoins et attentes; une de proposition de solution théorique, qui a engendré la rédaction d'un référentiel de grammaire à l'usage des enseignants, publié en 2011; une d'observation de terrain, toujours en cours; et une à venir d'intégration au terrain par l'insertion des propositions dans un nouveau curriculum.

2 Ce sont les observations qui nous ont permis de réaliser l'ampleur du problème de l'enseignement de la langue française à l'école et ont aidé à prendre conscience de ce que l'enjeu dépasse largement la seule préoccupation scientifique. Nous y reviendrons. D'autres constats ont également accéléré notre réflexion comme les résultats de l'enquête Program for international student ASSESSMENT (PISA) de 2009 - confirmés en $2013^{1}$ - qui, en plus de montrer de piètres performances en compréhension de l'écrit des élèves francophones, illustrent le fossé qui sépare la communauté française de Belgique des deux autres ${ }^{2}$. Il y a aussi, par exemple, les résultats des étudiants de première année de bachelier à l'examen de grammaire en première session ( $21 \%$ de réussite en $2010 ; 24 \%$ en 2011 au cours de Maitrise et critique de la grammaire normative de Dan Van Raemdonck à l'Université libre de Bruxelles) ou encore, même si cela n'a rien de probant, les chiffres de plusieurs rapports de l'Organisation de coopération et de développement 
économiques (OCDE) qui montrent un intérêt tout relatif des élèves pour l'école, notamment, et peut-être en particulier, dans les pays où l'on parle français. En Belgique, par exemple, jusqu'à un élève sur cinq scolarisés dans l'enseignement général ne trouve aucune utilité à l'école. Même s'il n'est pas responsable de ces chiffres, on ne peut pas dire que c'est l'enseignement de la grammaire qui donne le plus aux élèves le sentiment d'utilité de l'école.

\section{Les missions de l'école}

Nous avons voulu mesurer, par la présente recherche, dans quelle proportion les missions de l'école, quand elles passent par le filtre de l'établissement scolaire, la classe, d'un certain niveau de scolarité, par le cours de français et précisément de grammaire... mesurer, donc, à quel point ces missions sont finalement rencontrées. En 1997, la Communauté française - désormais Fédération Wallonie-Bruxelles (FWB) - a décidé de repréciser ses missions dans un décret éponyme. On peut y lire qu'il faut amener l'élève à la confiance en soi, lui apprendre à apprendre, le préparer à être un citoyen responsable et lui fournir la chance d'une émancipation sociale. Quant au programme d'enseignement du primaire, il charge, sans surprise, le cours de grammaire d'une mission de maitrise de la langue par et pour la communication. Éduscol - Portail national des professionnels de l'éducation (2013) précise, en caractères gras, les objectifs de l'école primaire française en des termes comparables:

Faire de l'école un lieu de réussite, d'autonomie et d'épanouissement pour tous, un lieu d'éveil à l'envie et au plaisir d'apprendre, à la curiosité intellectuelle, à l'ouverture d'esprit.

Dessein similaire formulé de façon détaillée dans la Déclaration de la Conférence intercantonale de l'instruction publique de la Suisse romande et du Tessin (CIIP), relative aux finalités et objectifs de l'École publique du 30 janvier 2003, notamment réflexion, collaboration, communication, démarche critique et pensée créatrice. Le Programme de formation de l'école québécoise (2006: 3), enfin, décline sa mission en trois volets: instruire, socialiser, qualifier, dont le deuxième est développé comme suit :

L'école doit être un agent de cohésion : elle doit favoriser le sentiment d'appartenance à la collectivité, mais aussi l'apprentissage du « vivre ensemble ». Dans l'accomplissement de cette fonction, l'école doit être attentive aux préoccupations des jeunes quant au sens de la vie. Elle doit promouvoir les valeurs qui fondent la démocratie et préparer les jeunes à exercer une citoyenneté responsable. Elle doit aussi prévenir en son sein les risques d'exclusion qui compromettent l'avenir de trop de jeunes.

5 Nous avons alors jugé pertinent d'observer si la démarche du cours de grammaire participait effectivement à rencontrer les missions de l'école, celles-ci étant presque identiques d'un pays à l'autre.

Il ne sera donc pas question ici de revenir et critiquer les missions de l'école, même s'il y a matière à le faire et que d'autres ne s'en sont pas privés. Brièvement. P. Perrenoud (2008 : 11), par exemple, reproche aux systèmes éducatifs de ne pas être en phase avec la «vie des gens "; ou M. Crahay (2000: 196) pour qui l'idéal égalitaire est tout à fait illusoire ; ou encore B. Rey et al. (2006: 24) qui résument la mission de l'école à l'acquisition de compétences ; ce que nuancent A. Barrère et D. Martuccelli (1998: 667) déjà avant eux en précisant que l'école doit tourner l'élève vers la collectivité plutôt que vers lui-même. 


\section{La rétroaction négative}

\section{Corpus}

7 Si notre intérêt de recherche porte d'abord sur l'observation de classes dans la perspective de mesurer la plus-value de l'introduction d'un nouveau référentiel de grammaire, nous faisons ici reposer nos observations sur un corpus d'interactions professeur-élève récoltées en 18 mois, entre septembre 2011 et avril 2013, soit 67 échanges de rétroactions négatives issus de 54 cours de grammaire de troisième et quatrième primaires (élèves de 8-9 ans). Voici un échantillon illustratif ${ }^{3}$ :

[Sur le genre du mot « professeur »]

P : «Professeur », c'est toujours au masculin, même pour les dames. Moi, je suis un

professeur.

$\mathrm{E}:$ Donc on fait une croix dans les deux?

$P$ : Non, on dit mon prof, ma prof, mais pas ma professeur.

[Sur les types de phrases]

$\mathrm{P}$ : Donnez-moi une phrase interrogative.

E : T'es riche?

$\mathrm{P}:$ Pardon?

$\mathrm{E}:$ Est-ce que tu es riche?

$P:$ Je préfère une autre.

$\mathrm{E}:$ Es-tu riche?

$\mathrm{P}$ : Voilà.

[Sur les déterminants]

Encadré : « Le déterminant a le même genre et nombre que le nom qu'il détermine ".

$\mathrm{E}:$ Ça veut dire quoi, « il détermine »?

$P$ : Ça veut dire « auquel il se rapporte ».

[Sur les démonstratifs]

$\mathrm{E}$ : Mais en fait, il y a des choses qui ne se montrent pas (ex : le cri).

$\mathrm{P}:$ Oui, ... c'est un peu compliqué... " se » est souvent tout près d'un verbe et " ce " tout près d'un nom.

[Sur l'impératif]

$\mathrm{P}:$ Quel conseil donnerais-tu à quelqu'un qui achète un nouveau gsm ?

E : Souvent, on dit d'aller dans...

E2 : Il faut faire attention à...

$P$ : Non, je veux juste le conseil.

E : ...

$\mathrm{E}:$ Va dans le magasin...

[Sur l'analyse]

Le professeur indique aux élèves comment trouver le sujet :

P : Il faut dire " c'est ... qui + verbe " ou " ce sont ... qui + verbe » ou poser la question « qui est-ce qui + verbe».

$\mathrm{P}$ : Le sujet est le mot qui fait l'action du verbe.

[Les élèves, qui comprennent toutes les phrases, ne voient pas pourquoi il faut trouver ce mot et l'appeler sujet.]

Nous reviendrons ensuite sur la définition, mais nous pouvons déjà avancer que ce sont des exemples de rétroactions négatives, c'est-à-dire, dans un premier temps, des retours correctifs sur une production :

1. le premier exemple montre un cas où l'élève accepterait deux propositions là où l'enseignant n'en autorise qu'une seule ; 
2. le deuxième illustre la préférence, voire l'attente, du professeur pour certaines propositions ;

3. le troisième concerne la terminologie utilisée et la manière qu'a le professeur de l'expliciter ;

4. le quatrième extrait montre comment l'enseignant réagit à l'incompréhension de l'élève relativement à l'explication donnée ;

5. en cinquième lieu, l'enseignant attend une réponse précise ;

6. et enfin, en dernier exemple, il donne une explication avec laquelle les élèves ne savent que faire.

\section{Définition d'une notion}

9 La notion de rétroaction est bien connue de la psychologie du développement et des sciences de l'éducation. Si l'on considère ces 45 dernières années, elle apparait sous différentes formes et vocables chez nombre d'auteurs. Chez G. L. Wyatt, le feedback (correctif) concerne la correction dans le cadre de l'acquisition du langage.

In acting as speech model for a child, the interested adult frequently will improve upon the child's speech performance. When Lisa (observation 4) talked about a " hangaroo ", her mother replied that Lisa would make a good " kangaroo ", thus providing the child with a corrective feedback in the area of articulation or phonics. (Wyatt, $1969: 14$ )

10 E. L. Deci et R. M. Ryan, qui ont beaucoup travaillé sur la question de la motivation, dégagent trois types d'évènements externes qui affectent la motivation : l'informationnel, le contrôleur et le démotivant, les deux derniers étant plutôt de type négatif. On est donc bien dans le cadre de la motivation en situation d'apprentissage.

These events are the informational, the controlling, and the amotivating events. Informational events are those that allow the individual to feel competent and self-determining. Such events promote intrinsic motivation. [...]. On the other hand, controlling events undermine the individual's sense of self-determination. They instil external control and diminish intrinsic motivation. [...]. Finally, amotivating events are events that underscore the individual's sense of incompetence. Because individuals come to feel incompetent, their intrinsic motivation for the activity is decreased. Negative performance feedback represents a powerful amotivating event which decreases intrinsic motivation. (Valleirand \& Reid, $1988: 240$ )

11 Chez P. Perrenoud, le feedback est un simple message qui affecterait le fonctionnement mental en vue d'un meilleur apprentissage.

Un feedback est un simple message. Pourquoi aiderait-il à apprendre ? Parce que l'apprenant en tient compte, parce qu'il affecte son fonctionnement cognitif. Les théories de la communication nous enseignent que l'efficacité d'un message se mesure au niveau de son destinataire : une intervention, une information n'aident un élève à mieux apprendre que si elles modifient ses processus cognitifs. Façon abstraite de dire que nul n'apprend à la place du sujet. On ne peut que stimuler, renforcer, réorienter, recadrer, accélérer son fonctionnement mental, dans l'espoir d'infléchir ses processus d'apprentissage. (Perrenoud, 1997)

C. Germain \& H. Séguin ajoutent le caractère implicite et explicite de la rétroaction, mais elle reste assimilée à de la correction dans le cadre d'un apprentissage de langue étrangère :

Une rétroaction négative explicite est définie comme toute rétroaction qui mentionne expressément le fait que les productions d'un apprenant sont non conformes à la langue cible. Une rétroaction négative implicite comprend toute 
correction de la forme erronée ainsi que les demandes de confirmation, les incompréhensions et les demandes de clarification, car dans chacun de ces cas, l'apprenant doit déduire que, s'il y a correction, c'est qu'il y a effectivement eu erreur. (Germain \& Séguin, $1998: 151$ ) ce qui est pareil, même si sous d'autres vocables chez J.-P. Cuq \& I. Gruca, mais trouve à se généraliser chez J. Lohisse pour qui la rétroaction est de l'ordre du comportemental et affecte tout l'acte communicatif.

L'attention à la forme a posteriori est plus compatible avec une approche communicative, mais elle demande beaucoup de disponibilité et de capacités chez le maître. L'avantage de cette solution est qu'on travaille sur le discours produit par l'apprenant, et qu'on est donc, au moins en apparence, plus centré sur lui. (Cuq \& Gruca, $2002: 349$ )

Il est possible d'introduire dans cette distinction les notions de rétroaction positive et rétroaction négative. Rappelons que, dans le feed-back positif, il y a évolution, transformation, changement, destruction du système alors que, dans le feed-back négatif, il y a homéostasie, amortissement du processus, retour à l'équilibre pour préserver ou rétablir le système. (Lohisse, 2006 : 166).

De manière synthétique, nous dirions que, pour notre part, la rétroaction négative est un retour correctif, plus ou moins explicite, verbal ou non, sur la production, verbale ou non, d'autrui.

\section{Changement de point de vue}

15 À la différence du point de vue de type « enseignant » adopté jusqu'à présent, qui place la rétroaction négative à l'origine d'un effet sur l'apprentissage, nous avons voulu analyser les effets qui seraient induits par une rétroaction négative envisagée comme outil dans un cadre d'apprentissage posé (point de vue de l'élève). En d'autres termes, quels sont les effets d'un apprentissage qui génère des rétroactions négatives? Pour ce faire, nous avons travaillé sur 67 échanges, comme précisé précédemment.

\section{Typologie des rétroactions négatives}

Nous pourrions classer nos occurrences dans des typologies existantes, dont la plus récente et complète serait celle de F. Georges \& P. Pansu (2011 : 109), mais, d'une part, elle se place du côté de l'enseignant, d'autre part, elle nous parait réductrice car tous nos échantillons seraient répartis en deux catégories du type "c'est faux » ou "c'est mal » parmi les feedbacks dits « simples». 
Tableau 1. Différentes formes de réactions possibles au comportement de réalisation ou à la performance d'un élève

\begin{tabular}{|c|c|c|c|c|c|}
\hline \multicolumn{3}{|c|}{ Feedback positif } & \multicolumn{3}{|c|}{ Feedback négatif } \\
\hline \multirow{2}{*}{$\begin{array}{l}\begin{array}{c}\text { Feedback } \\
\text { assertif }\end{array} \\
\text { Simple } \\
\text { type } 1\end{array}$} & \multicolumn{2}{|c|}{ Feedback évaluatif } & \multirow{2}{*}{$\begin{array}{c}\begin{array}{c}\text { Feedback } \\
\text { assertif }\end{array} \\
\text { Simple } \\
\text { type } 1\end{array}$} & \multicolumn{2}{|c|}{ Feedback évaluatif } \\
\hline & $\begin{array}{l}\text { Simple Complexe } \\
\text { type } 2 \text { estimatif }\end{array}$ & $\begin{array}{l}\text { Complexe } \\
\text { comparatif }\end{array}$ & & $\begin{array}{l}\text { Simple Complexe } \\
\text { type } 2 \text { estimatif }\end{array}$ & $\begin{array}{l}\text { Complexe } \\
\text { comparatif }\end{array}$ \\
\hline \multicolumn{6}{|c|}{ Exemples } \\
\hline $\begin{array}{l}\text { "C'est } \\
\text { juste. " }\end{array}$ & $\begin{array}{l}\text { "C'est " Tu as } \\
\text { bien." fait des } \\
\text { efforts." }\end{array}$ & $\begin{array}{l}\text { "Tu es } \\
\text { meilleur que } \\
\text { les autres." }\end{array}$ & $\begin{array}{l}\text { "C'est } \\
\text { faux." }\end{array}$ & $\begin{array}{l}\text { "C'est " Tu n'as } \\
\text { mal." pas fait } \\
\text { assez } \\
\text { d'efforts. " }\end{array}$ & $\begin{array}{l}\text { "Tu es } \\
\text { moins bon } \\
\text { que les } \\
\text { autres." }\end{array}$ \\
\hline
\end{tabular}

Note. Le caractère complexe (versus simple) du feedback renvoie à la contextualisation de la performance et le caractère évaluatif (versus assertif) réfère à la valeur sociale transmise par le feedback.

17 Nous avons préféré décomposer l'échange en empruntant le point de vue de la cible de la rétroaction, non de son origine ou de son objectif. Les éléments qu'il est alors possible d'isoler sont : l'énoncé, qui peut rester identique, disparaitre ou être changé ; le locuteur ; la proposition; et la rétroaction. Même s'il aurait pu sembler plus pertinent de figurer la rétroaction dans le sens inverse, nous avons préféré cette représentation pour une meilleure lisibilité de l'effet de celle-ci.

Figure 1. Éléments de figuration de la rétroaction

\begin{tabular}{|l|l|}
\hline Énoncé & Locuteur \\
\hline & \\
\hline Proposition & \\
\hline & Rétroaction \\
\hline
\end{tabular}

18 Sur cette base, nous avons envisagé toutes les combinaisons pertinentes possibles :

1. la proposition de l'élève qui est laissée sans suite ou délibérément écartée. Nous avons qualifié ce type d'«arbitraire» ou «dichotomique» quand l'exclusion repose sur le seul principe du vrai-faux ; 
Figure 2. La rétroaction par l'arbitraire

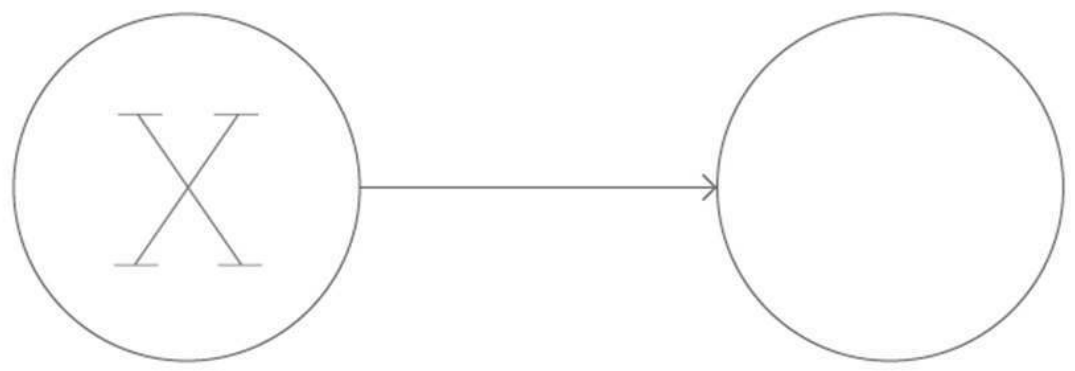

1. l'élève propose un énoncé que l'enseignant garde mais voudrait différent et conforme à son appréciation. Il s'agit du jugement esthétique ; du on ne dit pas x mais $x$;

Figure 3. La rétroaction par le jugement esthétique

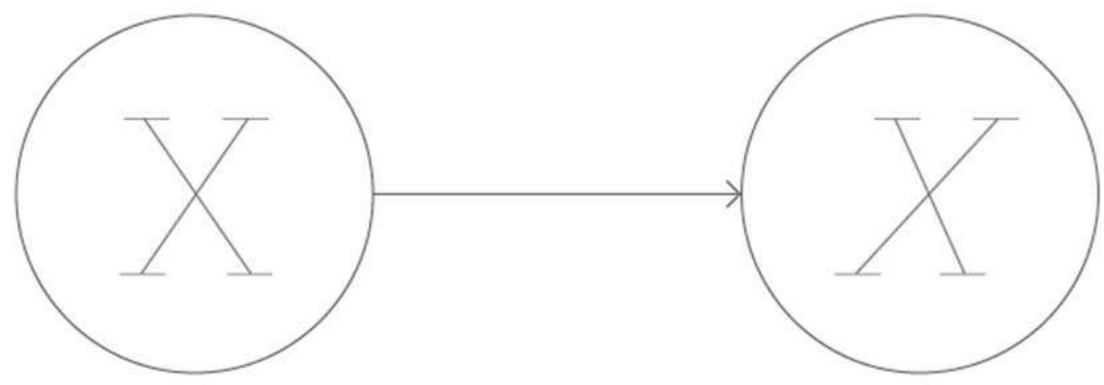

1. à la suite de sa proposition, l'élève la voit réduite sur le plan théorique ou lui-même dans sa qualité d'individu ; 
Figure 4. La rétroaction par la réduction

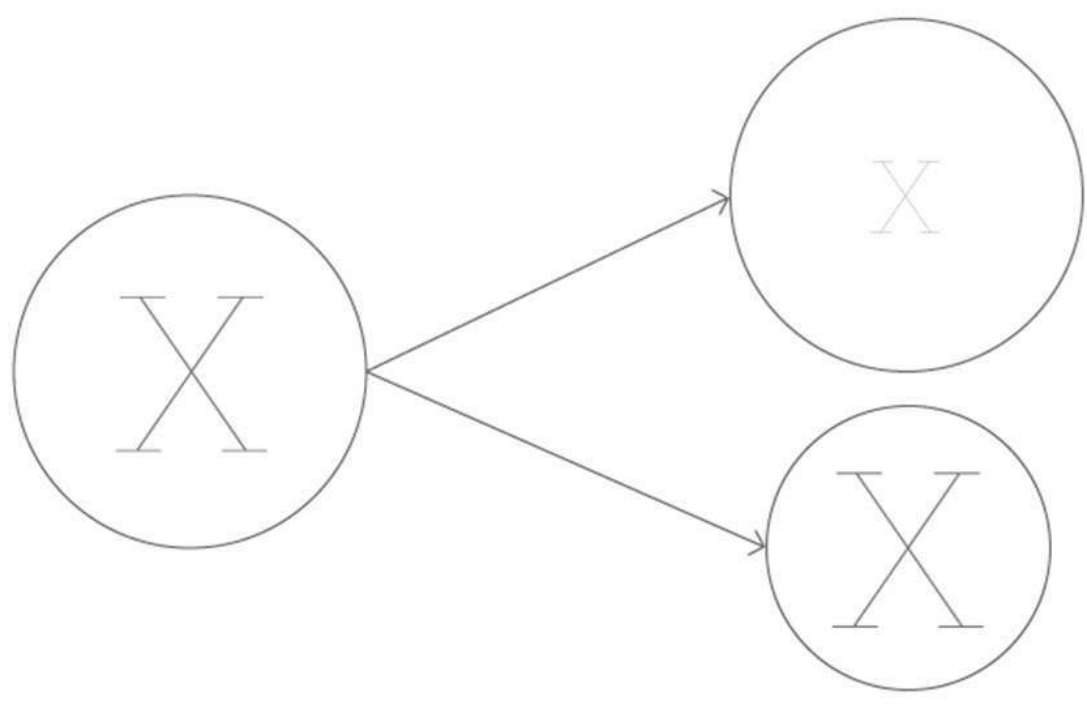

1. la proposition de l'élève se voit détournée ou altérée par l'enseignant ;

\section{Figure 5. La rétroaction par le détournement}

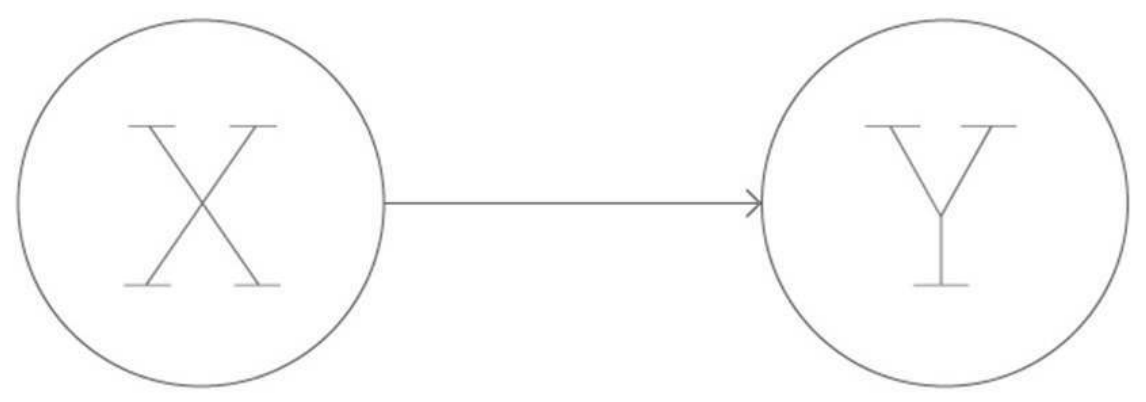

1. il se peut aussi que l'enseignant guide à un point tel ce qu'il attend que la proposition de l'élève n'est plus que le résultat de la rétroaction ou une proposition qui n'est pas le fruit de son intuition ; 


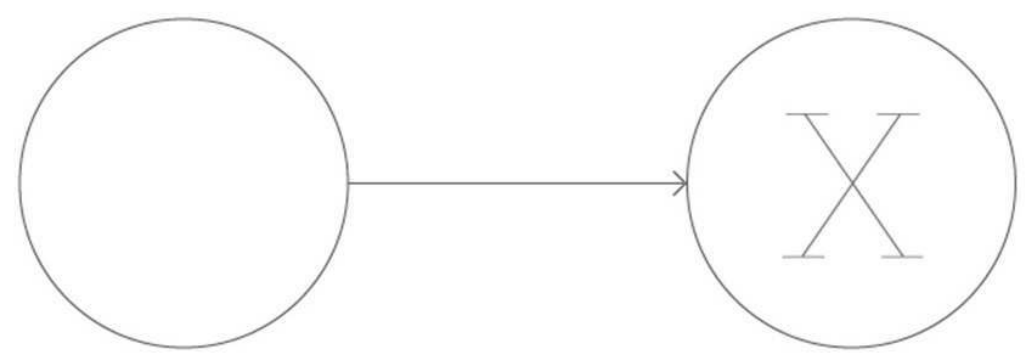

1. enfin, mais l'on sort quelque peu du registre de la rétroaction, l'enseignant peut faire intégrer par l'élève une proposition qui n'émane en rien de lui et dont il ne perçoit donc pas le sens. Ce type de rétroaction, dit démotivant, enlève autant la raison d'être de l'activité qu'elle ne suscite de motivation.

Figure 7. La rétroaction par dé-motivation

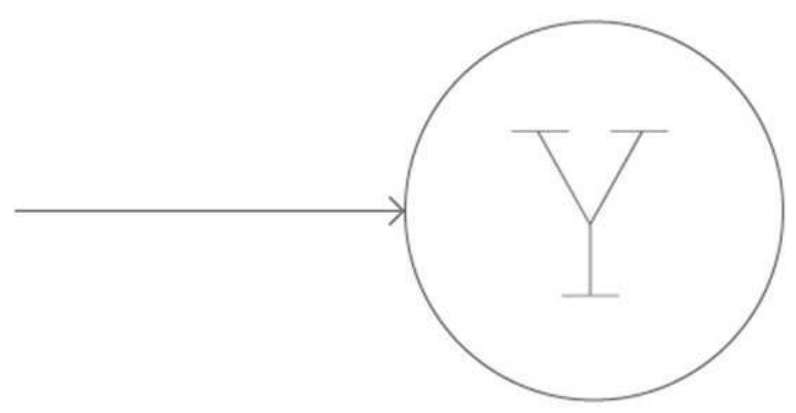

19 Les six exemples cités en entame de notre contribution correspondent chacun à l'un de ces types de rétroaction :

1. en 1, l'enseignant, arbitrairement, refuse le classement proposé par l'élève au nom de la dichotomie habituelle vrai-faux véhiculée par la grammaire traditionnelle et il n'accepte qu'une seule appartenance possible ;

2. en 2 , c'est un jugement de valeur, un jugement esthétique, qui exclut les propositions de l'élève. Les règles de l'écrit sont appliquées, implicitement, à l'oral ;

3. en 3 , l'enseignant réduit la théorie à l'évidence et ne rencontre pas l'attente de l'élève, amené à s'en satisfaire ;

4. en 4 , l'enseignant répond à une autre question que celle que lui posait l'élève, évitant cette dernière par la même occasion ;

5. en 5, il rejette les propositions correctes des élèves tant que ce n'est pas celle qui est attendue, forçant dès lors l'induction ou allant à l'encontre de leur intuition ;

6. enfin, en 6 , il est attendu des élèves qu'ils repèrent le sujet de la phrase mais ils ne comprennent pas pour quelle raison dans la mesure où ils perçoivent le sens. 
À classer tous les éléments de l'échantillon, nous pouvons même observer que $63 \%$ des occurrences, soit 42/109, appartiennent à plus d'un type figuré ci-dessus. Par exemple :

[Trouver le déterminant]

"Les trois coups ».

$\mathrm{E}:$ «les ».

E2 : heu, c'est pas « trois»?

P : Non, c'est « les ».

E2 : Et « trois », c'est faux?

P : Oui, ici c'est « les » car il n'y a pas « trois » tout seul.

Cet exemple illustre l'arbitraire en ce qu'il montre l'exclusion sans explication de la proposition de l'élève et le dichotomique parce que l'enseignant procède par vrai-faux et n'accepte qu'une seule réponse; la réduction théorique face à l'élève en demande d'explications ; la contre-intuition car il s'oppose à l'intuition des élèves, voire l'induction forcée car l'enseignant attend une solution précise comme seule possible; la démotivation car la discussion qu'il suscite éloigne du sens plus qu'il n'en rapproche.

\section{Effets de la rétroaction négative sur l'élève}

Nous prenons de nombreux raccourcis, mais les effets sur l'élève sont au moins de deux types. D'une part, il y a l'aliénation à une institution dépersonnalisante :

Autrement dit, la tension n'est autre que celle qui oppose, comme dans bien d'autres domaines de la vie moderne, l'individualisme expressif à une institution dépersonnalisante. C'est pourquoi, à bien des égards, les lycéens ont moins le souci de se voir octroyer de nouveaux droits, que le désir d'être mieux traités par les enseignants et par l'administration, et reconnus dans leur travail et dans leur personne. (Ballion, 1998) [...] Ces versions de l'« injustice scolaire » étant pour l'instant impossibles à traduire en termes de demandes collectives, l'engagement civique est alors fortement limité par le souci de l'authenticité juvénile. Il est toujours hypothéqué par les formes de négation de soi induites par les résultats scolaires. (Barrère \& Martuccelli, 1998 : 662).

Que dire alors de toutes ces rétroactions négatives qui participent, elles aussi à la "négation de soi ", déjà impliquée par les résultats scolaires, au détriment de l'engagement civique. D'autre part, la distanciation. Pour illustrer cette dernière, nous empruntons le continuum de B. Gerde \& M.-C. Bloch (2009) parce qu'il figure, en la simplifiant peut-être abusivement, l'évidente relation entre sentiment d'implication et décrochage.

Tableau 2. Le décrochage scolaire

Image 2000000C0000478800000EFB499F5E3A.wmf

\section{À qui la faute?}

Du fait des rétroactions négatives que nous avons observées, il y a tout lieu de craindre que les élèves se situent dans les trois colonnes les plus à droite ou qu'ils y tendent. Mais à qui la faute ? Peut-on raisonnablement incriminer l'enseignant? Il ne nous semble pas.

Si l'on se penche quelques instants sur les programmes disciplinaires eux-mêmes, on peut facilement repérer une série d'incohérences dont, par exemple le cas du sujet. 
Tableau 3. L'enseignement de la notion de sujet ${ }^{4}$

Le cas du sujet dans les programmes du primaire belge

2 Repérer les pronoms personnels sujets (à vivre dans l'action concrète)

2 Éviter les répétitions qui alourdissent en utilisant les substituts grammaticaux : un pronom personnel sujet.

Le groupe nominal sujet (GNS) et le groupe verbal (GV) forment la structure de base de la phrase.

En début de cycle, oralement, les questions de réflexion aboutiront à la reconnaissance du groupe nominal sujet.

2 En fin de cycle, le découpage de la phrase s'organisera autour de deux grandes questions qui aboutiront à la découverte des deux constituants : le groupe nominal sujet et le « reste de la phrase ".

le mot sujet à l'intérieur du GNS

3 Il marquera cette relation bilatérale par une flèche. Les moyens (critères) de reconnaissance du verbe et du sujet seront progressivement employés.

2

Reconnaissance du groupe sujet

La fonction sujet (GNS)

3

Découvrir : le verbe de la phrase; le sujet de la phrase / Associer : verbe et sujet; attribut (sans l'appeler par son nom) et sujet.

Son appartenance [attribut $\mathrm{du}$ sujet] est complexe: situé derrière le verbe, il qualifie pourtant le sujet; en fait, l'attribut « actualise » le sujet.

4 Ici, le sujet est un mot qui n'a pas réalisé l'action.

Le groupe du sujet sera isolé en répondant à la question : De qui... De quoi... parle-t-on dans

2 la phrase ? et en employant la forme emphatique «c'est... qui... ce sont... qui... »

Le mot sujet sera identifié à l'intérieur du GNS en montrant: qu'on ne peut pas le supprimer ;

3 qu'on peut le remplacer par un pronom; qu'en changeant le nombre de ce mot, le verbe aussi doit changer (choisir des variations audibles).

4 le complément d'agent : c'est le sujet de la forme active.

3 L'attribut du sujet : s'accorde avec le sujet.

2 Accorder le sujet et le verbe.

3 Le sujet est un être animé

4 Le sujet est un être inanimé 

primaire, il en résulte une définition du sujet du type :

La fonction sujet (GNS ou mot du GNS) peut être revêtue par un pronom personnel (substitut grammatical), un groupe - nominal ou non - et un mot de ce groupe, qui représentent un être animé ou non. Quand il forme un groupe nominal, il est l'un des deux constituants de base de la phrase, l'autre étant le groupe verbal, dont la fonction n'est pas à préciser. Il accorde l'attribut du sujet et s'accorde avec le verbe. De ce fait, si ce mot - pronominalisable et non suppressible - change en nombre, le verbe variera également. C'est un mot, qualifié et actualisé par l'attribut, qui réalise ou non l'action et devient complément d'agent à la voix passive. Il y a deux moyens de le trouver, soit par une question (De qui... De quoi... parle-t-on dans la phrase ?), soit en employant la forme emphatique (« c'est... qui... ce sont... qui... »).

27 C'est incompréhensible et chargé de contradictions : qu'est-ce que le sujet ? que peut-il être? Quel est son rôle? Une fonction peut-elle s'accorder? Avec une classe de mots (le verbe) ou avec une autre fonction (l'attribut du sujet)?

Et si l'on regarde de plus près l'accord de l'attribut du sujet dans les programmes de la francophonie du Nord, il s'observe rapidement que la question est traitée à 8,9 , voire 10 ans. Ce n'est pas grave, en soi... mais pourquoi est-ce le cas ? N'y a-t-il en matière de langue aucune progression logique et scientifique possible? L'exemple de la phrase négative est plus frappant encore car l'écart extrême peut être de quatre ans entre élèves pourtant voisins. Et devons-nous préciser que les élèves maitrisent cette notion bien avant même d'être scolarisés?

\section{L'enseignant, victime lui aussi}

Ce qu'il y a avec l'enseignant, c'est qu'il doit souffrir ces illogismes d'un discours disciplinaire duquel il se sent, lui aussi, toujours plus à l'écart et sur lesquels il n'a plus aucune emprise tant la grammaire est bien ancrée comme paradigme culturel. Il n'est bien souvent plus que pédagogue, relégué du côté de l'élève, bien loin de pouvoir s'autoriser la moindre prise sur le discours qu'il enseigne, le moindre recul. En effet, sur une échelle de construction du savoir, il intervient en dernière ligne avant l'apprentissage effectif.

Monde + être sensible $=$ expérience

Expérience + scientifique $=$ théorie

Théorie + enseignant-chercheur = savoir disciplinaire

Savoir disciplinaire + didacticien $=$ savoir scolaire

Savoir scolaire + pédagogue = enseignement

Enseignement + élève $=$ apprentissage

Et c'est le discours grammatical qui l'y pousse, lui dont C. Vargas (2009: 29) pointe toute l'incohérence.

La grammaire n'est pas obtenue par transposition didactique de savoirs savants, mais par recomposition de savoirs hétérogènes, certains obtenus à partir de la linguistique par emprunts-modifications, d'autres conservés de la grammaire scolaire déjà-là. Par recomposition, nous entendons que ces savoirs issus d'horizons très différents, ne sont pas véritablement articulés, synthétisés, mais sont juxtaposés, alors même qu'ils peuvent être inconciliables entre eux.

31 C'est parce qu'il est contre-intuitif que s'impose à l'enseignant une approche par déduction, du fait de son opacité terminologique qu'il se doit d'être explicite et, 
finalement, à cause de cette incohérence qu'il est poussé à l'intransigeance pour tenir une classe en révolte légitime.

\section{Conclusion}

En l'état, le cours de grammaire ne rencontre en aucun point les objectifs que l'école se fixe. Pire encore, il va à leur encontre en véhiculant les valeurs inverses de celles attendues. Nous l'avons vu avec l'exemple de la rétroaction négative. Or, «comme la vie scolaire n'est que le germe de la vie sociale, comme celle-ci n'est que la suite et l'épanouissement de celle-là, il est impossible que les principaux procédés par lesquels l'une fonctionne ne se retrouvent pas dans l'autre »(Durkheim, 1922: 40). Si la première victime est, assurément, sans doute inconsciemment, l'élève lui-même, l'enseignant ne doit pas en être tenu pour responsable, ou pour seul responsable. Ses rétroactions doivent compenser toutes les incohérences des programmes et progressions. C'est le discours grammatical en vigueur qui est pour majeure partie responsable. Il impose le choix didactique; l'enseignant est presque tenu de procéder par déduction et d'enseigner explicitement les points grammaticaux. Enfin, les incohérences le poussent à l'intransigeance. Dès lors, comment responsabiliser l'enseignant qui use de rétroactions négatives abusives? Pour autant, comment les accepter quand il est communément admis que du rapport à la norme en classe dépendent « la nature du lien social et les modes de socialisation à l'œuvre non seulement au sein de l'école, mais plus largement dans la société » (LeVasseur, Duval, 2013:42)?

\section{BIBLIOGRAPHIE}

BARRÈRE, A. \& MARTUCCELLI, D. (1998). « La citoyennete à l'école : vers la définition d'une problématique sociologique ». Revue française de sociologie 39 (4), p. 651-671.

Conseil des ministres de l'Éducation (2008). « Éléments d'un continuum langagier (Fondement et éléments d'un continuum)». Montréal : Chenelière, p. 1-34.

CRAHAY, M. (2000). L'École peut-elle être juste et efficace? De l'égalité des chances à l'égalité des acquis. Bruxelles: De Boeck.

- (2006). « Dangers, incertitudes et incomplétude de la logique de la compétence en éducation ». Revue française de pédagogie 154, p. 97-110.

CUQ, J.-P. \& Gruca, I. (2002). Cours de didactique du français langue étrangère et seconde. Grenoble : Presses universitaires de Grenoble.

DECI, E. L. \& RYAN, R. M. (1985). Intrinsic Motivation and Self-Determination in Human Behavior. New York : Plenum Press.

Conférence intercantonale de l'instruction publique de la Suisse romande et du Tessin (CIIP) (2003). Déclaration de la Conférence intercantonale de l'instruction publique de la Suisse romande et du 
Tessin (CIIP), relative aux finalités et objectifs de l'École publique. En ligne : www.ciip.ch/documents/ showFile.asp?ID=2521 (consulté le 09/12/13).

DEWEY, J. (2001). Democracy and Education, vol. 7. Penn State Electronic Classics Series, p. 368. En ligne : http://www.naturalthinker.net/trl/texts/Dewey,John/Dewey,_John__Democracy_And_Education.pdf (consulté le 09/12/13).

DURKHEIM, É. (1922). Éducation et sociologie. Éd. par J.-M. Tremblay, p. 51. En ligne : http:// content.yudu.com/Library/A1016u/education/resources/40.htm (consulté le 10/02/13)

Éduscol - Portail national des professionnels de l'éducation (2013). Présentation de l'école primaire. En ligne : http://eduscol.education.fr/cid46787/l-ecole-primaire.html (consulté le 09/12/13).

Fédération Wallonie-Bruxelles (2009). Programme des études 2009 pour l'enseignement fondamental. Belgique : Fédération Wallonie-Bruxelles.

GEORGES, F. \& PANSU, P. (2011). « Les feedbacks à l'école : un gage de régulation des comportements scolaires ». Revue française de pédagogie 176, p. 101-124.

GERDE, B. \& BLOCH, M.-C. (2009). « De l'inappétence au décrochage : quel processus ? Que proposer en amont et en aval ? ». La République des idées. En ligne : http://repid.com/De-l-inappetence-audecrochage.html (consulté le 09/02/13)

GERMAIN, C. \& SÉGUIN, H. (1998). Le Point sur la grammaire. Paris : Clé international.

Gouvernement du Québec, ministère de l'Éducation (2006). Programme de formation de l'école québécoise. En ligne : http://www1.mels.gouv.qc.ca/sections/programmeFormation/pdf/ prform2001nb.pdf (consulté le 11/11/13).

LEVASSEUR, L. \& DUVAL, S. (2013). «Vers une "socialisation démocratique" des élèves du primaire? ». Formation et profession 20 (1), p. 41-57.

LOHISSE, J. (2006). La Communication : de la transmission à la relation. $2^{2}$ éd. revue et augmentée par A. Klein. Bruxelles : De Boeck.

Ministère de l'Éducation nationale (2008a). « Cycle des apprentissages fondamentaux Progressions pour le CP et le CE1 ». Bulletin Officiel 3, hors-série, 19 juin. En ligne : http:// www.education.gouv.fr/bo/2008/hs3/apprentissages.htm (consulté le 15/04/13).

- (2008b). «Cycle des approfondissements - Progressions pour le CE2 et le cours moyen ». Bulletin officiel 3, hors-série, 19 juin.

Ministère de la Communauté française (1997). Décret définissant les missions prioritaires de l'enseignement fondamental et de l'enseignement secondaire et organisant les structures propres à les atteindre. En ligne : http://www.gallilex.cfwb.be/fr/leg_res_01.php?ncda=21557\&referant=101 (consulté le 09/02/13)

Organisation de coopération et de développement économiques (OCDE) (2013). Résultats du PISA 2012: Savoirs et savoir-faire des élèves : Performance des élèves en mathématiques, en compréhension de l'écrit et en sciences. Paris : PISA/Éditions OCDE.

PERRENOUD, P. (1996). « Le dialogue scolaire, un échange définitivement inégal ?». Revue de psychologie de la motivation 21, p. 116-123.

- (1997). « De l'évaluation formative à la régulation maitrisée des processus d'apprentissage. Vers un élargissement du champ conceptuel ». En ligne : http://www.unige.ch/fapse/SSE/ teachers/perrenoud/php_main/php_1997/1997_11.html (consulté le 05/06/13).

- (2008). Préface. In: Audigier, F. \& Tutiaux-Guillon, N. (éds), Compétences et contenus : les curriculums en questions. Bruxelles : De Boeck, p. 7-14. 
République et Canton de Genève, Département de l'Instruction publique (2007). Plan d'études de l'enseignement primaire : $1 \mathrm{E}-6 \mathrm{P}$.

ReY, B., CARETTE, V., DEFRANCE, A. \& Kahn, S. (2006). Les Compétences à l'école. Apprentissage et évaluation. $2^{\mathrm{e}}$ éd. Bruxelles : De Boeck.

ROBINSON, K. (2010). « Changing Education Paradigms ». RSA Animate [trad. Simon Lapointe]. En ligne : http://www.ted.com/talks/ken_robinson_changing_education_paradigms.html (consulté le $01 / 01 / 13)$.

RYAN, R. M. \& DECI, E. L. (2000). «Intrinsic and Extrinsic Motivations : Classic Definitions and New Directions ». Contemporary Educational Psychology 25 (1), p. 54-67.

VAN RAEMDONCK, D., et al. (2004-2013). GRAMM-R scola I et II. Différents rapports sur l'analyse du discours grammatical remis à la FWB. Bruxelles : Université libre de Bruxelles.

- (2011). Le Sens grammatical. Référentiel à l'usage des enseignants. Bruxelles: Peter Lang.

VARGAS, C. (2009). « Peut-on inventer une grammaire pour la réussite scolaire? ». Repères 39, p. 17-39.

WYATT, G. L. (1969). Language learning and communication disorders in children. New York : Free Press, p. 372.

\section{NOTES}

1. Les résultats de l'enquête Pisa de 2012 (Pisa, 2013) placent toujours les jeunes belges francophones sous la moyenne de l'Organisation de coopération et de développement économiques (OCDE) et en bons derniers de la francophonie du Nord.

2. De surcroit, au niveau du retard scolaire, la Communauté française compte $44,3 \%$ d'élèves qui ne sont pas à l'heure et la France 35,5\%.

3. Où $P$ indique une prise de parole du professeur, $E$ de l'élève et les gras la rétroaction négative.

4. La première colonne indique le cycle concerné par cet enseignement (cycle 2:5-8 ans; cycle 3 : 8-10; cycle $4: 10-12$ ans). Le programme est celui de l'enseignement organisé par la Fédération Wallonie-Bruxelles.

\section{RÉSUMÉS}

Dans cette étude, centrée sur les premières années de l'enseignement de la grammaire française (langue maternelle), nous avons tenu à montrer le poids de la rétroaction négative, c'est-à-dire du retour correctif, plus ou moins explicite, verbal ou non, sur la production, verbale ou non, de l'élève. Le discours grammatical scolaire traditionnel regorge de contradictions qui sont propices au questionnement des élèves. Or, il n'en est fait que peu de cas, pour divers motifs allant de l'inconfort de l'enseignant aux contraintes du manuel, ce qui implique de fréquents recadrages. Le modèle descriptif, en six étapes, des différentes rétroactions négatives observées que nous dégageons permet d'indexer tous les échanges de ce type et d'illustrer à quel point nous sommes en droit de craindre que les activités proposées durant ces cours - et les rétroactions négatives 
qu'elles impliquent - ne participent pas à l'autonomisation des élèves et à la construction de leur esprit critique, deux missions pourtant essentielles de l'école.

In this study, focused on the first years of the French grammar courses for native speakers, we demonstrated the importance of negative feedback. The traditional grammar language for school abounds with contradictions conducive to questioning by the pupils. However, their questions are not taken into account for reasons ranging from discomfort of the teacher to the constraints of the textbook. We propose a descriptive model in six stages - based on our observations - that allows us to index all exchanges of this type. The model further enables us to illustrate and document our fear that the activities proposed during the course - and the negative feedbacks they imply - do not contribute to the empowerment of students and the construction of critical thinking, two essential missions of the educational system.

\section{INDEX}

Mots-clés : pédagogie, rétroaction, grammaire française, enseignement, motivation

Keywords : pedagogy, feedback, french grammar, education, motivation

\section{AUTEUR}

\section{LIONEL MEINERTZHAGEN}

Ladisco, Plateforme internationale GRAMM-R, Université libre de Bruxelles (ULB) 